\title{
Is Piezomagnetism Influenced by Microcracks during Cyclic Loading?
}

\author{
Randolph J. MARTIN III \\ Department of Geosciences, The Pennsylvania State University, \\ University Park, Pennsylvania, U.S.A.
}

(Received July 23, 1980)

\begin{abstract}
Before magnetic field variations in tectonically active regions can be used to infer changes in the crustal stress distribution, it must be demonstrated that piezomagnetism is a stress dominated rock property. Changes in many rock properties of geophysical interest such as electrical conductivity and seismic wave velocity during compressional loading are dominated by changes in microcrack porosity. If piezomagnetism were to fall into such a category its applicability as a method for determining in situ stress fluctuations would be limited. In order to assess the influence of microcrack dilatancy on magnetic susceptibility and remanent magnetization a series of cyclic loading experiments were conducted at confining pressures up to $200 \mathrm{MPa}$. The results of these tests indicate that piezomagnetism is a stress related property, that is, it is controlled by differential stress and only marginally, if at all, affected by changes in microcrack porosity. Magnetic susceptibility decreased with increasing differential stress. The change in susceptibility was uniquely related to differential stress and totally independent of confining pressure, the number of stress cycles or the magnitude of the inelastic volumetric strain. Remanent magnetization, on the other hand, showed a pronounced demagnetization on the first cycle which rapidly decreased on subsequent cycles as long as the peak cycle stress remained constant. An increase in peak stress caused a pronounced decrease in magnetization at the termination of the cycle. There is a striking similarity between this observation and the change exhibited in volumetric strain; that is, an increase in crack porosity at the termination of the first cycle that became progressively smaller with increasing cycle number. Both remanent magnetization and volumetric strain became reproducible on the same cycle. In spite of this apparent correlation other test suggest that such results are merely fortuitous and changes remanent magnetization during cyclic loading are dominated by differential stress.
\end{abstract}

\section{Introduction}

Local changes in the magnetic field prior to earthquakes are well documented (TAZIMA, 1966; IPSIR and UyAR, 1971; SMITH and Johnston, 1976). These changes 
ranged from 1 to $40 \gamma$ and they appear to be definitely real. For example, Smith and JOHNSTON (1976) observed a $1.5 \gamma$ increase in the total magnetic field about seven weeks before the 1975 magnitude 5.2 Thanksgiving Day earthquake near Hollister, California. The anomaly persisted for two weeks and then returned to its initial value. In addition to precursory observations co-seismic changes in magnetization have also been reported during the 1946 Nandaido earthquake (KATO and UTASHIRO, 1949), and the Matsushiro swarm (RikitaKe, 1968). Furthermore, the Cannikin explosion produced a $7 \gamma$ increase in the magnetic field along a fault $1.6 \mathrm{~km}$ from the detonation (HasbroucK and Allen, 1972).

One possible interpretation of these observations is that perturbations of the stress field in the epicentral region produced detectable variations in the magnetization of rocks prior to and during the event. In fact, existing laboratory data show that magnetic susceptibility and remanent magnetization exhibit a pronounced stress dependence.

While existing theories of piezomagnetism are for the most part based on magnetostriction, rocks in tectonically active regions can deform inelastically due to the development of microcrack dilatancy. Since most of the early piezomagnetic measurements did not correlate changes in either remanent magnetization and magnetic susceptibility with the volume strain of the sample, the possibility existed that piezomagnetism was a crack related property in the same way as electrical resistivity and seismic wave velocity. That is, during deformation variations in a crack related property are predominantly due to variations in crack porosity and less dependent on the stress required to generate the porosity.

It is important to determine whether piezomagnetism is a stress dominated or crack dominated property because only if it is stress related can it be used to satisfactorily correlate changes in rock magnetism with tectonic stress. In order to assess the relative contribution of microcrack dilatancy on the changes in remanent magnetization and magnetic susceptibility during stress cycling a laboratory study was initiated. The relation between remanent magnetization, stress, volumetric strain and confining pressure has been presented previously (MARTIN et al., 1978). Based on those results it was concluded that remanent magnetization was a stress dominated property but dependent to some extent on microcrack dilatancy. In light of this it seemed worthwhile to refine the estimate of the contribution of crack initiation and propagation on remanent magnetization as well as determine its effect on magnetic susceptibility. With these problems in mind a series of experiments were carried out.

\section{Experimental Procedure}

The magnetic susceptibility and remanent magnetization experiments described in this paper were carried out on the Ralston intrusive from Golden, Colorado. This rock was selected for illustrative purposes from five different rock types that have been 


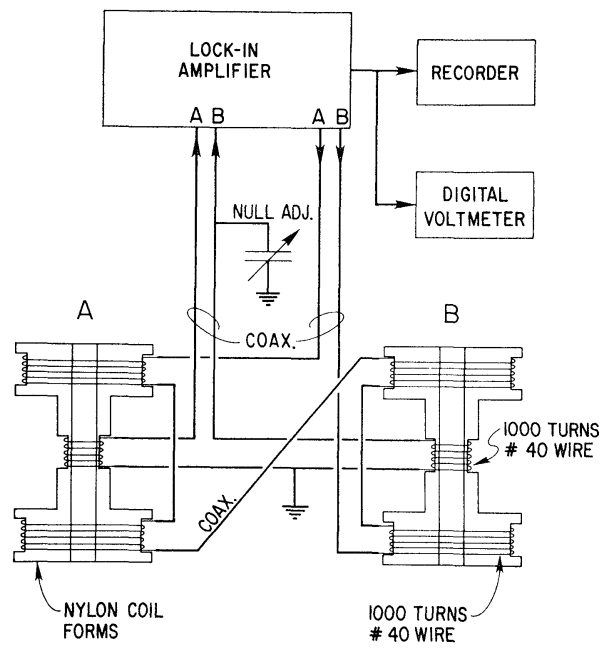

Fig. 1. Schematic diagram of the apparatus for measuring axial susceptibility.

studied in detail. The Ralston intrusive is an unaltered diabase with a modal analysis of $36 \mathrm{Or}, 28 \mathrm{An}_{41}, 23 \mathrm{Au}, 4 \mathrm{Ol}, 2 \mathrm{Bio}, 1 \mathrm{Ap}$ and $6 \mathrm{Ox}$. The mean grain diameter is $0.7 \mathrm{~mm}$ and the porosity is 0.003 . The titanomagnetite grains range in size from 20 300 microns and appear homogeneous under an optical microscope. However, upon examination under the SEM at 10,000X the grains looked mottled with thick ilmenite lamellae on (111). The Curie temperature of the Ralston intrusive is $535^{\circ} \mathrm{C}$.

All the tests were carried out on ground, right circular specimens $1.52 \mathrm{~cm}$ in diameter and $4.12 \mathrm{~cm}$ in length. Each experiment was run in a beryllium copper pressure vessel bolted into a hydraulic loading frame. A typical experiment consisted of step wise loading the sample to 90 to $95 \%$ of its fracture strength and then unloading. Each stress increment was approximately $20 \mathrm{MPa}$. At each stress level the axial force, axial and circumferential strain and either the axial susceptibility or the axial and radial components of the remanent magnetization were measured. The confining pressure was continuously monitored with a $200 \mathrm{MPa}$ Heise gauge.

The susceptibility measurements were carried out in a bridge apparatus modified after the design of CHRISTIE and Symons (1969). Figure 1 is a schematic diagram of the susceptibility bridge. The bridge was a transformer balance in which the internal oscillator of a Princeton Applied Research lock-in amplifier (Model 124A with model 116 differential preamplifier) was used to excite the primary coils of the measuring transformer and the reference transformer. The net in-phase signal picked up by the two secondary coils, connected in series opposition, was returned to the amplifier input where it passes through a transformer, a linear amplifier, a narrow-band filter, a phasesensitive detector for comparison with the output phase and finally to a d.c. voltmeter 
and a strip chart recorder. Each primary coil consisted of two Helmholtz coils, with 1,000 turns of No. 40 enameled wire wound on a nylon form $5.40 \mathrm{~cm}$ in diameter, separated by $4.13 \mathrm{~cm}$ and driven at a frequency of $1,000 \mathrm{~Hz}$ with the PAR output adjusted to generate a field of $0.5 \mathrm{Oe}$. The secondary coil, positioned in the center of the coaxial Helmholtz coils had 1,000 turns of No. 40 enameled wire wound on a diameter of $3.00 \mathrm{~cm}$.

The axial susceptibility is determined by first balancing the output of the secondary coils and then inserting the sample into the axial cavity of one nylon coil form. The sample generates an imbalance in the output of the secondary coils which is proportional to the susceptibility of the sample. The bridge was calibrated with a series of powders containing various percentages of titanomagnetite grains. The low field susceptibility of these powders was determined by the National Bureau of Standards, Boulder, Colorado.

With the sample emplaced, one sensing head was inserted into the pressure vessel while the reference coil assembly remained outside the pressure vessel. The vessel was pressurized to the desired confining pressure, the secondary coils were once again balanced and then cyclic deformation tests were carried out. The amount of imbalance during loading and unloading is a measure of the change in axial susceptibility.

Samples were prepared for each susceptibility test in the following manner. Two strain gages were epoxied to the rock specimen, one axial and one circumferential. The sample was then wrapped with a thin sheet of teflon to reduce the friction between the jacket and the strain gages, and inserted in a polyurethane jacket. Two lucolux spacers were positioned to keep conductive material away from the end of the sample. The jacket was then sealed by epoxying hardened steel plugs to the open ends of the jacket.

The changes in remanent magnetization during cyclic loading were measured using the same technique described by MARTIN et al. (1978). For these tests the pressure vessel was enclosed in a two layer mu metal shield. Changes in magnetization during stress cycling were monitored with two pairs of flux gate magnetometers. One pair of magnetometers was arranged to detect the axial and radial component of the remanent moment while the other pair was placed in an identical configuration about a beryllium copper dummy sample to monitor changes in the background field within the pressure vessel. The change in remanent magnetization was obtained by taking the difference between the reading at the sample with the appropriate background level.

A TRM was induced in each sample of the Ralston intrusive by first heating the specimen to $600^{\circ} \mathrm{C}$ and then cooling it in a 1.0 Oe field with the field axis inclined to the sample axis. After measuring the remanent intensity and the inclination of the vector with respect to the axis of the sample, both the rock and the beryllium copper dummy were encased in a copper jacket. Strain gauges were epoxied to the jacket to measure the axial and circumferential strain of the sample. The sample was inserted 


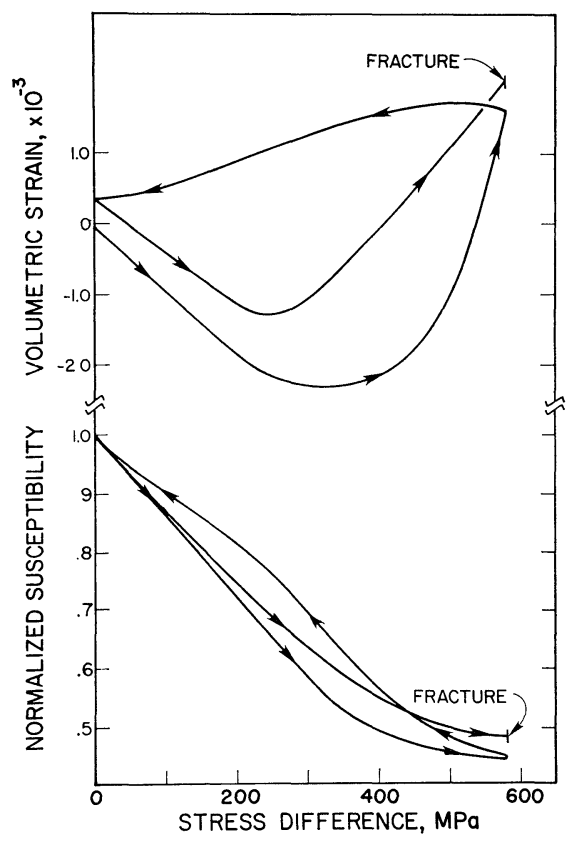

Fig. 2. Effect of cyclic stress on axial magnetic susceptibility. Normalized changes in magnetic susceptibility and volumetric strain are plotted as a function of differential stress for the Ralston intrusive tested at $50 \mathrm{MPa}$ confining pressure. The data represents the first complete cycle; the sample was fractured on the second cycle. The initial susceptibility was $4.6 \times 10^{-3} \mathrm{emu} / \mathrm{cm}^{3}$-Oe.

in the magnetometer assembly so that the magnetic vector was in the plane of the magnetometers. The sample and assembly were then pressurized and cyclically loaded.

\section{Experimental Results}

\subsection{Magnetic susceptibility}

The results of a typical experiment on the Ralston intrusive at a confining pressure of $50 \mathrm{MPa}$ are shown in Fig. 2. Normalized axial susceptibility and volumetric strain are plotted as a function of differential stress. As the sample was loaded the susceptibility decreased. At approximately $230 \mathrm{MPa}$ the susceptibility vs stress curve became strongly nonlinear and its slope decreased perceptibly. At the peak stress of $580 \mathrm{MPa}$, the susceptibility had decreased by $56 \%$. There was a slight hysteresis on unloading and no permanent demagnetization at the completion of the cycle. The rock was brought to failure on the second cycle. The shape of the susceptibility curve for the second cycle was similar to that of the first cycle except the change in susceptibility was somewhat less. Furthermore there was no precursory change in suscepti- 


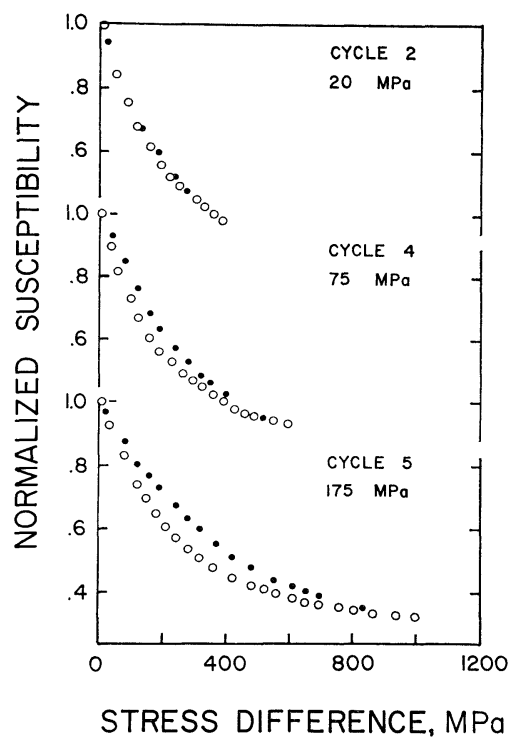

Fig. 3. Change in normalized susceptibility as a function of differential stress for a sample of Ralston intrusive. Cyclic experiments were carried out at successive confining pressures of 20,75 , and $175 \mathrm{MPa}$. Open circles indicate loading; solid dots indicate unloading. The initial susceptibility was $4.2 \times 10^{-3} \mathrm{emu} / \mathrm{cm}^{3}-$ Oe.

bility just prior to failure.

At first glance, the results in Fig. 2 may suggest that there is a correlation between the onset of pronounced dilatancy and a perceptible change in the slope of the susceptibility vs stress curve. For the Ralston intrusive this region is in the vicinity of 300 $\mathrm{MPa}$ differential stress at $50 \mathrm{MPa}$ confining pressure. In order to determine whether or not the flattening of the susceptibility curve above $300 \mathrm{MPa}$ or so was a direct consequence of increasing crack porosity several experiments were carried. If the break in the slope of the susceptibility curve in Fig. 2 is related to the onset of dilatancy then as the confining pressure is increased the shape of the curve should remain the same but span greater stress range since larger stresses will be required to achieve the same inelastic volumetric strain. Conversely, if susceptibility is independent of microcrack dilatancy and only a function of differential stress then the stress sensitivity factor will be constant regardless of the confining pressure. The results of one experiment on the Ralston intrusive are shown in Fig. 3. Three cycles were carried out at a confining pressure of $20 \mathrm{MPa}$ (only the second cycle is shown); the pressure was then augmented to 75 and $175 \mathrm{MPa}$ successively and the fourth and fifth cycle run. At $20 \mathrm{MPa}$ confining pressure, the susceptibility decreased by $60 \%$ as the differential stress was increased to $400 \mathrm{MPa}$. The change in susceptibility was not linear; the rate of change decreased with increasing stress. Upon unloading there was vitually no 
hysteresis. Similarly at 75 and $175 \mathrm{MPa}$ confining pressure the slope of the susceptibility vs stress curve decreased over its entire loading path. In fact, the curve appears to flatten out and asymptotically approach a saturation value of $37 \%$ of the initial susceptibility at a differential stress of $900 \mathrm{MPa}$. The loading curves for the three cycles shown are nearly identical and are superposable. All exhibit a nearly $60 \%$ decrease at $400 \mathrm{MPa}$ differential stress, for example. The unloading curves however, are not superposable. It appears that the hysteresis upon unloading increases as the peak differential stress for the cycle is increased.

The validity of this type of experiment, where the confining pressure is varied from cycle to cycle, can be justified in two ways. First, there is no permanent demagnetization at the termination of a cycle and second, the effect of confining pressure on susceptibility is minimal (Fig. 7). The change in initial susceptibility between 0 and $150 \mathrm{MPa}$ was less than $2 \%$, appreciably smaller than the changes observed during nonhydrostatic loading.

The results of the experiments carried out on the other four rocks studied in detail were very similar to those reported here for the Ralston intrusive. Specifically the loading curves were reproducible, dependent predominantly on differential stress, exhibited a saturation at nearly $40 \%$ of the initial susceptibility at about $900 \mathrm{MPa}$ differential stress and showed a progressively larger hysteresis upon unloading as the peak differential stress for the cycle was increased. Furthermore, little or no change in susceptibility was observed as the confining pressure was augmented from 0 to $200 \mathrm{MPa}$.

\subsection{Remanent magnetization}

An experiment similar to the one described to obtain the data presented in Fig. 3 was carried out to determine to what extent remanent magnitization is a stress related

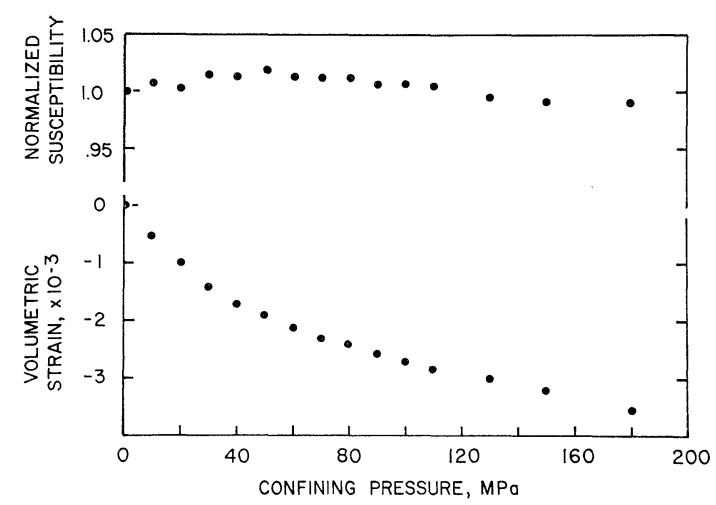

Fig. 4. Change in normalized susceptibility and volumetric strain as a function of confining pressure only for a sample of Ralston intrusive. The initial magnetic susceptibility was $4.2 \times 10^{-3} \mathrm{emu} / \mathrm{cm}^{3}$-Oe. 


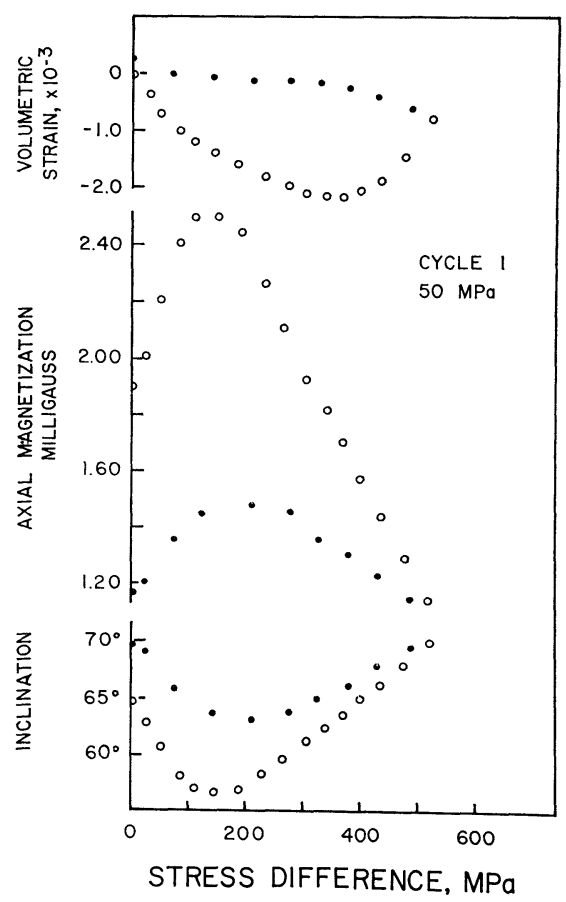

Fig. 5. The change in the axial component of remanent magnetization, the inclination of the remanent vector with respect to the loading axis and the volumetric strain are plotted as a function of differential stress for the fitst loading cycle at a confining pressure of $50 \mathrm{MPa}$. The specimen had a TRM with an initial intensity $4.1 \times 10^{-3} \mathrm{emu} / \mathrm{cm}^{3}$. Open circles indicate loading and solid dots unloading.

property. The results of an experiment with four stress cycles are shown in Figs. 5, 6,7 , and 8 . For each experiment the volumetric strain, axial component of the remanent vector, and the angle between the remanent vector and the sample axis are plotted as a function of differential stress. The first and second cycles were run at a confining pressure of $50 \mathrm{MPa}$ (Figs. 5 and 6). On the first cycle the specimen decreased in volume elastically until the stress exceeded $300 \mathrm{MPa}$, then the sample began to dilate. At a peak stress of $525 \mathrm{MPa}$ the sample was unloaded; there was a pronounced hysteresis in volumetric strain as the differential stress was removed from the sample. The axial component of the remanent vector increased $32 \%$ during initial loading to $140 \mathrm{MPa}$. With continued loading, the axial magnetization changed direction and started to decrease. At the peak stress for the cycle the magnetization had dropped to $60 \%$ of its initial value. Upon unloading there was a pronounced hysteresis and a permanent demagnetization of $38 \%$ at the termination of the cycle. The angle between the magnetic vector and the sample axis decreased from $64^{\circ}$ to $56^{\circ}$ as the stress was initially raised to $140 \mathrm{MPa}$. Then as loading progressed the magnetic vector 


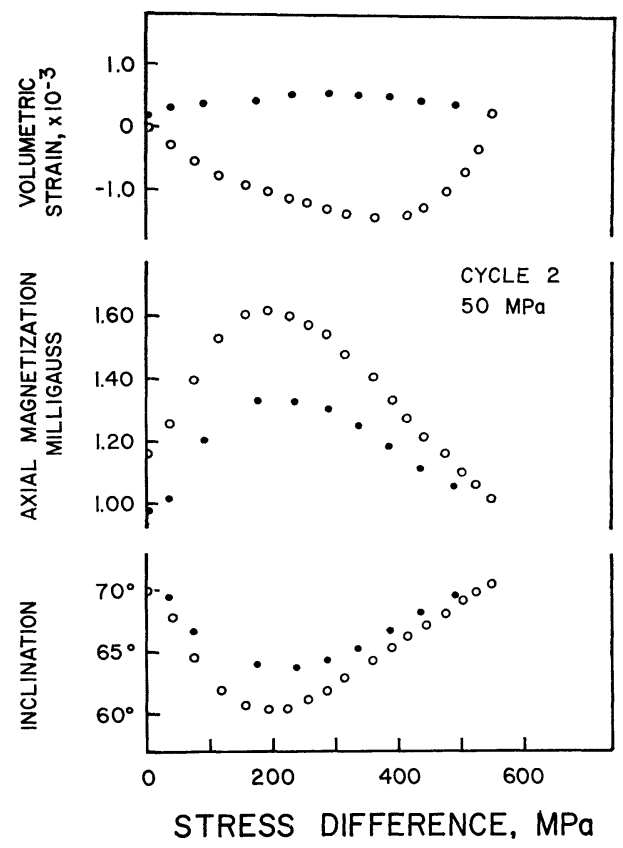

Fig. 6. The change in the axial component of remanent magnetization, the inclination of the remanent vector with respect to the loading axis and the volumetric strain are plotted as a function of differential stress for the second cycle of the Ralston intrusive sample shown in Fig. 5. The confining pressure was $50 \mathrm{MPa}$. Open circles indicate loading and solid dots unloading.

rotated away from the stress axis and at the peak stress level the angle had increased to $70^{\circ}$. There was a pronounced hysteresis during unloading and a permanent change in the orientation of the remanent vector. The second cycle (Fig. 6) was similar to the first except the permanent demagnetization at the completion of the cycle was only $6 \%$ and there was only a slight permanent change in the orientation of the remanent vector. For the third cycle (Fig. 7) the confining pressure was augmented to $100 \mathrm{MPa}$. The volumetric strain behaved as in the previous cycle except the onset of dilatancy increased to $420 \mathrm{MPa}$, there was a permanent increase in crack porosity at the end of the cycle. The axial magnetization increased by $28 \%$ as the specimen was loaded to $140 \mathrm{MPa}$. As deformation proceeded the axial magnetization began to decrease and at the peak stress of $674 \mathrm{MPa}$ was only $38 \%$ of its initial value. Unloading exhibited a marked hysteresis and a permanent demagnetization of $22 \%$. The variation in the inclination of the magnetic vector with respect to the stress axis was consistent with the first two cycles. The vector rotated toward the stress axis as the sample was stressed to $180 \mathrm{MPa}$ and then rotated toward the stress axis as the sample was stressed to $180 \mathrm{MPa}$ and then rotated away from the axis for the remainder of the 


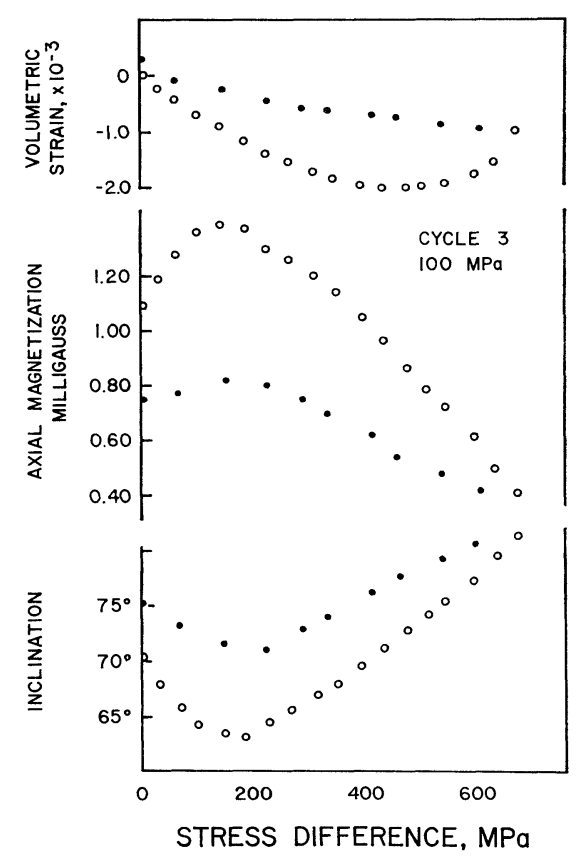

Fig. 7. The change in the axial component of remanent magnetization, the inclination of the remanent vector with respect to the loading axis and the volumetric strain are plotted as a function of differential stress for the third cycle of the Ralston intrusive sample shown in Figs. 5 and 6 . The confining pressure was $100 \mathrm{MPa}$. Open circles indicate loading and solid dots unloading.

loading cycle. There was a large hysteresis upon unloading and a permanent rotation of $5^{\circ}$ away from the stress axis at the completion of the cycle.

The fourth cycle (Fig. 8) was carried out at a confining pressure of $20 \mathrm{MPa}$ and the loading cycle terminated with the fracture of the sample. The data obtained on this cycle was very similar to that for the preceeding cycle. The maximum increase in the axial component of the remanent magnetization occurred at $170 \mathrm{MPa}$ and then the magnetization decreased right through failure. There was no significant variation in magnetization that suggested failure was imminent. The magnetic vector rotated toward the stress axis until the differential stress reached $170 \mathrm{MPa}$ and the rotated away during the remainder of the loading path. There was no premonitory change in the inclination of the magnetic vector prior to failure of the sample.

\section{Discussion}

The experimental results presented here support the conclusion that changes in magnetic susceptibility and remanent magnetization during cyclic loading are pre- 


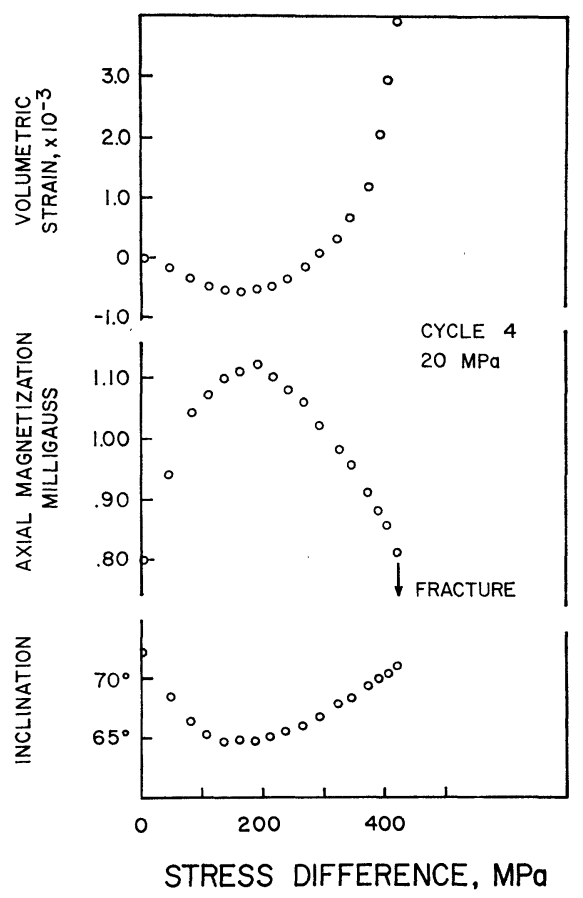

Fig. 8. The change in the axial component of remanent magnetization, the inclination of the remanent vector with respect to the loading axis and the volumetric strain are plotted as a function of differential stress for the fourth cycle of the Ralston intrusive sample shown in Figs. 5, 6, and 7. The confining pressure was $20 \mathrm{MPa}$. The sample failed at differential stress of $423 \mathrm{MPa}$.

dominantly due to variations in differential stress and are only marginally influenced by confining pressure and microcrack dilatancy. The susceptibility experiments are particularly convincing; for example the decrease in susceptibility with increasing stress was indentical over a range of confining pressures from 20 to $175 \mathrm{MPa}$ (Fig. 3). With increasing confining pressure, higher differentail stresses are required to achieve the same inelastic volumetric strain. If susceptibility was affected by changes in crack porosity then some perceptible change in the slope of the susceptibility vs stress curve should coincide with the onset of pronounced dilatancy and shift to progressively higher differential stresses with increasing confining pressure. No such shift is apparent in the data. Furthermore, changes in compressibility up to $200 \mathrm{MPa}$ or so are dominated by changes in crack porosity. Again if changes in crack porosity influence magnetic behavior then there should be some correlation between changes in volumetric strain during hydrostatic compression and magnetic susceptibility. There was no consistent change in susceptibility that correlated with the changes in volumetric strain (Fig. 4). 
The unloading hysteresis in the susceptibility curves are puzzling. As the peak differential stress of a test cycle increases the observable hysteresis during unloading increases. It is unlikely that this effect is related to changes in crack porosity. The magnitude of the hysteresis in the volumetric strain during unloading is to a first approximation independent of confining pressure (compare volumetric strain at 50 and $100 \mathrm{MPa}$ in Figs. 5, 6, and 7). Therefore, if there was a relation between volumetric strain and susceptibility, the unloading hysteresis should be of the same order of magnitude for all cyclic tests where the peak stress is between 90 and $95 \%$ of the fracture stress at that confining pressure. While a physical explanation of the increase in hysteresis of the magnetic susceptibility with increasing differential stress is not possible from this data, it does not seem to be related to the hysteresis in the volumetric strain curve.

Finally crack related physical properties such as electrical resistivity (BRACE and ORANGE, 1968) and seismic wave velocity (GUPTA, 1973; HolcomB, 1978) exhibit relatively modest changes as the rock is loaded through the elastic region. As microcrack dilatancy develops the changes become pronounced and vary in nearly direct proportion to the increase in crack porosity. As the sample approaches fracture the rate of change of such properties accelerate and can be used as precursors to rock failure. In contrast, the rate of change in magnetic susceptibility with increasing crack porosity showed no correlation and there was no precursory change just prior to failure. Revol et al. (1977) carried out uniaxial tests on magnetite and reported a precursory recovery in susceptibility just prior to failure. However the stress-strain data accompanying the experiment indicates the sample was loaded unevenly and subjected to bending. Therefore it is possible that the recovery prior to failure was an artifact of the way the sample was loaded.

While it can be convincingly shown that the behavior of magnetic susceptibility during cyclic compression tests is dominated by differential stress and independent of confining pressure and microcrack dilatancy, the relation between remanent magnetization, stress and inelastic volumetric strain is equivocal. MARTIN et al. (1978) found that there was a correlation between remanent magnetization and volumetric strain during cyclic loading. For these experiments the first cycle produced a large demagnetization and the volumetric strain exhibited a large increase in crack porosity. The hysteresis loops during unloading were greatest for both the magnetization and volumetric strain on the first cycle. With increasing cycle number progressively smaller hysteresis loops were observed and the demagnetization and the increase in porosity at the termination of the stress cycle diminished. By the fifth cycle both the magnetization and volumetric strain stabilized and became reproducible. Furthermore, consider the data presented in Figs. 6 and 7. At the end of cycle 2 at $50 \mathrm{MPa}$ confining pressure there was no permanent demagnetization or increase in crack porosity. When the confining pressure was augmented to $100 \mathrm{MPa}$ and the peak differential stress for cycle 3 increased, there was a permanent demagnetization and an in- 
crease in crack porosity at the termination of the cycle. Thus there seems to be at least some affect of microcrack dilatancy on remanent magnetization for large differential stresses.

However if the data of KEAN et al. (1976) is considered a completely different interpretation can be forwarded. KEAN et al. (1976) carried out cyclic uniaxial tests, with a peak stress of $40 \mathrm{MPa}$ on several rock types. An irreversible decrease in the remanent moment was observed on the first cycle; subsequent cycles were reversible. With a peak stress of less than $40 \mathrm{MPa}$ it is unlikely that microcrack dilatancy developed and thus the stress vs volumetric strain curve should be reversible. This leads to a situation where there is an unloading hysteresis in the remanent magnetization without a corresponding hysteresis in volumetric strain. In light of this it is possible that the correlation between the magnetization and volumetric strain reported here and by MARTIN et al. (1978) was merely fortuitous. More experimental work is required to resolve the problem.

Another way of assessing the contribution of inelastic volumetric strain to the piezomagnetic effect is to determine some identifiable break in the slope of the remanent magnetization curve that coincides with the onset of dilatancy at a fixed confining pressure and then see if that feature also coincides with the development of pronounced axial cracking at higher and lower confining pressures. The peak increase in the axial component of the remanent magnetization provides such a readily identifiable reference point. Thus, if microcrack dilatancy were responsible for the pronounced change in slope then this point should shift to higher differential stresses with increasing confining pressure as more stress will be required to initiate significant changes in crack porosity. An examination of Figs. 5, 6, 7, and 8 reveals that the stress corresponding to a zero slope in the remanent magnetization curve (and also approximately the same stress at which the magnetic vector ceased to rotate toward the stress axis and begins to rotate away from it) occurs at $170 \pm 20 \mathrm{MPa}$ for confining pressures of 20,50 , and $100 \mathrm{MPa}$. This observation implies that the change in the sign of the slope in the axial component of remanent magnetization vs stress curve is not related to cracking and the distribution of cracks within the rock but is primarily a function differential.

Based on the experimental results presented here, the variation in magnetic susceptibility and remanent magnetization during cyclic loading are stress related properties and only marginally, if at all influenced by changes in microcrack density. Obviously other factors such as composition, grain size, micro-structure of the grains, and type of remanent magnetization will affect the total piezomagnetic effect, but for each set of parameters there will be a corresponding stress response depending on the differential stress acting on the rock.

Since piezomagnetism is a stress dominated effect, changes in rock magnetism in tectonically active regions are directly related changes in the in situ stress field. Ideally then by measuring the total piezomagnetic effect in the laboratory variations in 
tectonic stress can be calculated from magnetic field measurements. An additional constraint on the absolute magnitude and orientation of the principal stresses can be obtained if hydrofracture in situ stress determinations have been previously conducted in the area. In this case a good estimate of the angular relation between the inducing field, remanent vector and axis of greatest compressive stress can be obtained. This not only aids in data reduction but also may permit absolute stress determinations.

Before magnetic changes can be used to directly infer stress, at least one remaining problem must be addressed. Large permanent decreases in remanent magnetization were observed at the termination of the first stress cycle for all the rocks studied. Such irreversible effects could introduce sizeable errors in any analysis. Most tectonically active regions have most likely undergone hundreds to thousands of stress cycles. Consequently, it may be prudent to ignore the demagnetization recorded for the first few cycles and consider only the reversible changes characteristic of the latter cycles. However, since a single stress cycle may take hundreds of years viscous remanent effect may make each recycling of an area look like the first cycle. The question then is to determine which remanent behavior is applicable, that of the first cycle or that of subsequent cycles. Further consideration must be given to this problem.

In conclusion, the total piezomagnetic effect is dominated by differential stress and only slightly, if at all, influenced by microcrack dilatancy.

This research was supported by the U. S. Geological Survey under contract No. 14-08-001-17702 and by the National Science Foundation, Division of Earth Sciences under grant No. EAR 7919534.

\section{REFERENCES}

Brace, W. F. and A. S. ORANGE, Electrical resistivity changes in saturated rocks during fracture and frictional sliding, J. Geophys. Res., 73, 1433-1445, 1968.

Christie, K. W. and D. T. A. Symons, Apparatus for measuring magnetic susceptibility and its anisotropy, Geol. Survey of Canada Paper, 69-41, 1969.

GuPTA, I. N., Seismic velocities in rock subjected to axial loading up to shear fracture, J. Geophys. Res., 78, 6936-6942, 1973.

Hasbrouck, W. P. and J. H. Allen, Quasi-static magnetic field changes associated with the Cannikin nuclear explosion, Bull. Seismol. Soc. Am., 62, 1479-1487, 1972.

Holcomв, D. J., A quantitative model of delatancy in dry rock and its application to Westerly granite, J. Geophys. Res., 83, 4941-4950, 1978.

ISPIR, Y. and O. UYAR, An attempt in determining the seismomagnetic effect in NW Turkey, $J$. Geomag. Geoelectr., 23, 295-305, 1971.

KATO, Y. and S. UTASHIRo, On the changes in the terrestrial magnetic field accompanying the great Nankaido earthquake in 1946, Sci. Rep. Tohoku Univ. Ser., 5, 40-43, 1949.

KeAN, W. R., R. DAy, M. Fuller, and V. A. Schmidt, The effect of uniaxial compression on the initial susceptibility of rocks as a function of grain size and composition of their constituent titanomagnetites, J. Geophys. Res., 81, 861-872, 1976.

Martin, R. J., R. E. HabermanN, and M. Wyss, The effect of stress cycling and unelastic volumetric strain on remanent magnetization, J. Geophys. Res., 83, 3485-3496, 1978.

Revol, J., R. DAY, and M. D. FulleR, Magnetic behavior of magnetite and rocks stressed to failure- 
relation to earthquake predictin, Earth Planet. Sci. Lett., 37, 296-306, 1977.

RIKITAKe, T., Geomagnetism and earthquake prediction, Tectonophysics, 6, 59-68, 1968.

SMITH, B. E. and M.J.S. Johnston, A tectonomagnetic effect observed before a magnitude 5.2 earthquake near Hollister, California, J. Geophys. Res., 81, 3556-3560, 1976.

TAZIMA, M., Accuracy of recent magnetic survey and locally anomalous behavior of the geomagnetic secular variation in Japan, Thesis, University of Tokyo, 1966. 